On the Hardness of Allocating FREQUENCIES FOR HYBRID NETWORKS by

Ewa Malesińska Alessandro Panconesi No. 498/1996 


\title{
On the Hardness of Allocating Frequencies for Hybrid Networks
}

\author{
Ewa Malesińska* \\ TU Berlin, MA 6-1 \\ Str. des 17 Juni 136 \\ 10623 Berlin, Germany \\ Email: malesin@math.tu-berlin.de
}

\author{
Alessandro Panconesi ${ }^{\dagger}$ \\ FU Berlin, Informatik \\ Takustr. 9 \\ 14195 Berlin, Germany \\ Email: ale@inf.fu-berlin.de
}

February 1996

\begin{abstract}
This paper studies the channel stability number, a combinatorial function that has been introduced for evaluating frequency allocation plans for hybrid cellular networks. We present several results concerning the approximability of this function in the case of complete graphs and analyze how different constraints influence its computational complexity.
\end{abstract}

\section{Introduction}

In this paper we study a combinatorial problem arising in the context of frequency allocation strategies for cellular networks. Before defining the problem and listing our results, it is perhaps best to give the relevant context so that the genesis and the relevance of the problem can be better appreciated.

In the frequency allocation problem for mobile telephone networks we are given a socalled interference graph and a frequency spectrum consisting of a set of carrier frequencies. Vertices in the graph represent stations, each requiring a certain number of frequencies. Two nodes in the graph are adjacent if the signals transmitted by the corresponding stations can interfere. For technical reasons, two frequencies $f$ and $g$ assigned to the same station must satisfy $|f-g|>\delta$, where typically $\delta=2$. Frequencies assigned to interfering stations must satisfy this inequality with $\delta=0$ or 1 . The optimization goal is to assign frequencies so that the expected traffic can be supported keeping at the same time the interferences low. This is formalized in several ways according to the context. The allocation strategies currently in use in all large, i.e. non local, networks follow a static approach: frequencies are assigned once for all. Reallocation happens rarely, usually every two or three months or when the network undertakes restructuring (stations are added, replaced or deleted). This static approach has several shortcomings [ZE'93]. In particular, it does not make a good use of the limited number of frequencies that are reserved for one cellular telephone network, a number usually in the order of 50. Consider for instance a typical metropolis like Berlin or New York

\footnotetext{
*Supported by the graduate school "Algorithmic Discrete Mathematics". The graduate school is supported by the Deutsche Forschungsgemeinschaft, grant WE 1265/2-1.

${ }^{\dagger}$ Supported by a research fellowship of the Alexander von Humboldt Foundation
} 
City; during the day, phone call traffic tends to be very heavy in the downtown area and light in the suburbs. In the evening the pattern is just reversed. Ideally, a network should be able to adapt to such changes and allocate frequencies dynamically as needed. In the above example the traffic pattern is predictable, but in reality this will not be the case; the network should be able to reconfigure dynamically according to (unpredictable) contingencies. No doubt this will be a common scenario in the next future and several dynamic allocation strategies have already appeared in the literature (see e.g. [DJLS'94, DV'93, EB'91, R'92]). Between the current situation and the appearance of dynamic networks however, there will be a transition phase where networks will be hybrid, i.e. partly dynamic and partly static. It is this type of networks that we are concerned with in this paper. A central problem arising when considering hybrid networks is the following: allocating frequencies to the static part of the network constraints the available frequency spectrum for vertices of the dynamic part. Such an assignment might be too restrictive and it is important to be able to detect whether this is the case. A related and rather important problem is to devise computationally efficient methods allowing to compare different frequency assignments to the static part of the network, i.e. to decide which of two assignments is the best with respect to the dynamic part.

Therefore, in this paper we define and study the following combinatorial problem. An input instance $I$ consists of an interference graph $G=(V, E)$ modeling the dynamic part of the network and, for each vertex $u$, a list of available frequencies $L(u)$ and two requirements $\max (u)$ and $\min (u)$ denoting the minimum and maximum number of frequencies required by the station corresponding to $u$. The $L(u)$ 's are subsets of some linear order $\mathcal{F}$ - the frequency spectrum- which is also part of the input $G$. The optimization goal is to select subsets $S(u) \subseteq L(u)$ in order to maximize

$$
\sum_{u \in V}|S(u)|
$$

subject to some or all of the following constraints: (a) for all $u, \max (u) \geq|S(u)| \geq \min (u)$; (b) for all $(u, v) \in E$, if $f \in S(u)$ and $g \in S(v)$ then $|f-g|>0$ (i.e. $S(u) \cap S(v)=\emptyset$ ); (c) [co-site constraint] for all vertices $u$, if $f \in S(u)$ and $g \in S(u), f \neq g$, then $|f-g|>1$. We call the maximum value of $\sum_{u \in V}|S(u)|$ the channel stability number of $I$ and denote it with $\operatorname{ch}(I)$.

The input subsets $L(u)$ model the fact that frequencies allocated to the static neighbors of $u$ are not available for $u$ any more. Constraint (a) models the fact that for dynamic stations the actual number of frequencies needed can vary unpredictably within two values. Although a station, strictly speaking, functions as long as there is one available channel, in practice each station, in order to service its area satisfactorily, requires a minimum number of channels. This parameter, denoted as $\min (u)$, is an estimate done by the network managers. Constraint (b) ensures that no two neighboring stations use the same frequency. In real systems, a stronger version of constraint (c) is required, namely $|f-g|>\delta>0$. Usually, $\delta=2$. The simpler condition $|f-g|>1$ does not affect the validity of our results except that approximation factors should be scaled down by a $\delta$ factor. The function $\operatorname{ch}(I)$ measures how much "flexibility" is left to the dynamic part of the network.

This problem simultaneously generalizes other well-known combinatorial problems related to vertex coloring: the T-coloring problem, list coloring, set coloring (a generalization of vertex coloring whereby one seeks to assign sets to vertices so that sets assigned to adjacent vertices have empty intersection) and the $k$-th stability number, also known as the partial k-coloring problem, where one seeks a vertex induced $k$-colorable subgraph of maximum size [B'89, H'80, JT'95, T'89]. 
In this paper, we study the channel stability number problem for the special case of cliques. The importance of this special case stems from several facts. First, graphs coming from real systems have the clique number bounded by a rather small constant, in the order of 15 or so. Such a condition is simply enforced by the managers of real networks who, in order to avoid severe signal interference, make sure that large cliques are never created. When a clique is too large it can be broken in several ways for instance, by decreasing the emission power so that stations sufficiently far apart cease to interfere. If, as a result, some regions are left underserviced new stations are introduced as appropriate. The net result of such operations is replacement of a large clique by two or more smaller ones. In this fashion the maximum degree is kept under control and usually is in the order of 20. Some old stations might have higher degrees- in the order of 50- but they are being gradually modified and their degree is expected to decrease. It should also be pointed out that the topology of these graphs, although unknown, is by no means arbitrary. In particular, it resembles intersection graphs of roughly disk shaped regions. Such graphs have several nice properties. In particular, their chromatic number is bounded by a constant times the clique number [MBHRR'95, GSW'94].

These considerations are corroborated by the experimental evidence available. When allocating frequencies telephone companies routinely compute all maximal cliques of the whole graph using backtracking algorithms. Such computations take the order of minutes even for the largest existing networks which have as many as 5,000 nodes [P'95]. Given that all maximal cliques are available studying complete graphs is all the more relevant. On the one hand, a given static allocation plan should pass the test of performing well on each clique. On the other, it is plausible that good upper estimates of $\operatorname{ch}(\cdot)$ for the whole network can be computed from the cliques.

In a previous paper, Malesińska showed that when constraint (c) is dropped the problem is solvable in polynomial time by reducing it to bipartite matching, and that when (c) is introduced the problem becomes non approximable in a rather strong sense (unless $\mathrm{P}=\mathrm{NP}$ ): to find any feasible solution satisfying (a) through (c) is NP-hard [M'95].

In this paper, we continue this line of research. After some preliminary definitions in Section 2, we study the approximation complexity of $\operatorname{ch}(I)$ in Section 3. Given that under constraints (a) through (c) even finding a feasible solution is NP-hard, we study the problem under relaxed constraints. First, we show that, interestingly, if any feasible solution is available then a 1/3-approximation can be computed in polynomial time. Then, we show that when constraint (a) is relaxed by dropping the set of conditions $|S(u)| \geq \min (u)$, for all $u$, the problem becomes 1/2-approximable. It cannot, however, be approximated to any degree of accuracy because, as we show, it is MAX SNP-hard- a fact which rules out the existence of polynomial-time approximation schemes for the problem, unless $P=N P$. In Section 4 we show that the approximation ratio of $1 / 2$ can be improved if the input instances satisfy a certain "sparsity" condition of the lists $L(u)$ of of admissible frequencies. Finally, in Section 5 we give some "density" conditions for these lists that, when satisfied, make the co-site constraints have no influence on the maximum value of $\operatorname{ch}(I)$.

\section{Preliminaries}

We recall some well-known notions and definitions from the theory of approximation algorithms (see e.g. [CLR'90, PY'91]). An NP maximization (minimization) problem $A$ is $\alpha$-approximable if there exists a polynomial time algorithm $\mathcal{A}$ which, for all inputs $I$, produces a solution $\mathcal{A}(I)$ whose value is at least (at most) $\alpha$ times the optimal value. We say 
then that the algorithm $\mathcal{A}$ has the performance guarantee $\alpha$. Alternatively, the quality of an approximation algorithm can be measured by its relative error, i.e.

$$
\max _{I} \frac{\left|o p t_{A}(I)-\mathcal{A}(I)\right|}{o p t_{A}(I)} .
$$

Note that if the relative error of an approximation algorithm $\mathcal{A}$ is bounded by $\epsilon$ then $\mathcal{A}$ has the performance guarantee $1-\epsilon$ if $A$ is maximization problem, and $1+\epsilon$ if $A$ is a minimization problem.

A problem $A L$-reduces to another problem $B$ if we can find two constants $\alpha$ and $\beta$ and a pair $(f, g)$ of polynomially computable functions such that: (a) for all instances $I$ of $A$, $f(I)$ is an instance of $B$ such that $\operatorname{opt}_{B}(f(I)) \leq \alpha \operatorname{opt}_{A}(I)$; and (b) given a feasible solution $b$ of $f(I)$ whose value is $c(b)$ then, $a=g(b, f(I))$ is a feasible solution of $I$ of value $c(a)$ such that $\left|o p t_{A}(I)-c(a)\right| \leq \beta\left|o p t_{B}(f(I))-c(b)\right|$. Taken together, these two conditions imply that if $B$ is approximable with worst case relative error $\epsilon$ then $A$ is approximable with the relative error $\alpha \beta \epsilon$ or, conversely, that if there exist a limit $\delta_{0}$ such that $A$ cannot be $\left(1-\delta_{0}\right)$-approximated (or $\left(1+\delta_{0}\right)$-approximated if $A$ is a minimization problem) then, $B$ cannot be approximated within $1-\delta_{0} \alpha^{-1} \beta^{-1}$ (or $1+\delta_{0} \alpha^{-1} \beta^{-1}$ ).

In a seminal paper, Papadimitriou and Yannakakis introduced a class of combinatorial problems called MAX SNP which has many natural complete problems with respect to L-reductions [PY'91]. Among these is MAX 3SAT-B whose input is a boolean formula $F\left(x_{1}, \ldots, x_{n}\right)$ in conjunctive normal form such that each clause has at most three literals and each variable appears at most B times. The optimization goal of MAX 3SAT-B is to find a truth assignment satisfying the maximum number of clauses. Recent breakthroughs in the theory of approximation algorithms show that if a problem $A$ is MAX SNP- hard w.r.t. L-reductions then there exist some $\delta_{A}$ such that $A$ cannot be $\delta_{A}$-approximated provided that $\mathrm{P} \neq \mathrm{NP}$ (see [A'94, BGS'93, H'94] among others).

As defined in the introduction ch $(I)$ denotes the channel stability number of an instance $I=(G, L, \min , \max )$ consisting of the interference graph $G$, lists of available frequencies $L(u)$ and minimum and maximum channel requirements. If no minimum frequency requirements have to be observed-or, equivalently, if $\min (u)=0$, for all $u$-than the corresponding channel stability number is denoted by $\widetilde{c h}(I)$. Analogously, if only conditions (a) and (b) are considered and the co-site constraints are ignored then the channel stability number is denoted by $\operatorname{ch}^{*}(I)$.

As customary, $K_{n}$ denotes the complete graph on $n$ vertices. In this paper, $\operatorname{ch}(I), \widetilde{c h}(I)$ and $c h^{*}(I)$ are studied when the underlying graph topology is a complete graph. Moreover, note that in this paper the notions of frequency and channel are used interchangeably as logical rather than technical terms and that they are often represented as colors.

\section{Approximability of the channel stability number}

In this section we show some results on the approximability of the channel stability number. It is an obvious necessary condition for the existence of a polynomial time approximation algorithm for any optimization problem that at least one feasible solution can be computed in polynomial time. However, the following theorem is proven in [M'95]:

Theorem 1 Consider the class of instances of ch $(I)$ defined on a complete graph and satisfying $\max (v)=\min (v)=r(v)$. Then, it is NP-complete to decide if there is a feasible solution, i.e. a collection of $S(u) \subseteq L(u)$ satisfying constraints (a) through (c). 
In view of this, it makes sense to study the approximability of the channel stability number only if we have some information about the feasibility of the instance. The next result shows that if at least one feasible solution exists then $\operatorname{ch}(I)$ is $\frac{1}{3}$-approximable.

Theorem 2 Consider an instance I of the channel stability problem defined on a complete graph $K_{n}$ and a frequency spectrum $C$. If the instance $I$ is feasible then $\operatorname{ch}(G)$ can be $\frac{1}{3}$ approximated in time $O(M n|C| \sqrt{M n+|C|})$. If additionally one feasible solution $S(u)$ is known for I then the algorithm yields not only the approximated value of $\operatorname{ch}(G)$ but also a new set of feasible sublists reaching this value.

\section{Proof.}

We claim that if there is at least one feasible solution for the instance $I$ satisfying all requirements (a) through (c) then $\operatorname{ch}(I) \geq \frac{1}{3} c h^{*}(I)$, where $c h^{*}(\cdot)$ denotes the number of channels that can be assigned when only conditions (a) and (b) are observed. On the other hand, $\operatorname{ch}(I) \leq c h^{*}(I)$. This yields a polynomial time approximation algorithm with the performance guarantee $\frac{1}{3}$ as $c h^{*}(I)$ can be computed in time $O(M n|C| \sqrt{M n+|C|})$ ([M'95]). Note that this algorithm does need to know any feasible solution for the instance $I$. Simply the knowledge of its existence is enough to assure the performance guarantee.

Now, in order to complete the proof we construct a solution $P$ by combining any feasible solution $S$ satisfying all constraints with a solution $S^{\prime}$ that selects $\operatorname{ch}^{*}(I)$ channels, but does not necessary observe the co-site constraints. The new assignment $\mathrm{P}$ satisfies all the constraints and $\sum_{v \in V}|P(v)| \geq \frac{1}{3} \operatorname{ch}^{*}(I)$. It is constructed in two stages. First, $\min (v)$ colors from the solution $S$ are assigned to each vertex $v \in V$. Let us consider these colors according to their linear order. When a channel $c$ is added to $P(v)$ then the set $S^{\prime}(v)$ is reduced to $S^{\prime}(v) \backslash\{c-1, c, c+1\}$. Moreover, if there is another vertex $w \in V$ such that $c \in S^{\prime}(w)$ then $c$ is removed from the list $S^{\prime}(w)$. Hence, in the first stage $\sum_{v \in V} \min (v)$ channels are introduced to the solution $P$ and at most three times as much elements are removed from the solution $S^{\prime}$.

Let us denote the remaining elements of the solution $S^{\prime}$ by $S_{r}^{\prime}(v), v \in V$. If now $S_{r}^{\prime}(v)$ satisfies the condition $\left\lceil\left|S_{r}^{\prime}(v)\right| / 2\right\rceil \leq \max (v)-\min (v)$ for all vertices $v \in V$ then in the second stage every second element of the lists $S_{r}^{\prime}(v)$ can be added to the solution $P$ and the proof is completed since the overall value of the solution $\sum_{v \in V}|P(v)|$ is at least $\frac{1}{3} \operatorname{ch}^{*}(I)$. Otherwise, let us assume that there is a vertex $w \in V$ such that $\left\lceil\left|S_{r}^{\prime}(w)\right| / 2\right\rceil=\max (w)-\min (w)+x$, for some $0<x \leq \min (w)$. It means that $\min (w)$ - the number of channels added to the list $P(w)$ from the list $S(w)$ - exceeded at least by $x$ the number of channels removed during the first stage from the list $S^{\prime}(w)$. That is there were at least $x$ channels in the list $S(w)$ added to $P(w)$ such that neither they were in $S^{\prime}(w)$ nor any of the neighboring channels had to be removed from $S^{\prime}(w)$ when they were added to $P(w)$. Some of these $x$ channels were only possibly removed from $S(v)$, for one vertex $v \neq w$ per channel. Therefore, since per one channel introduced to the solution $P$ we can afford deleting three channels from the solution $S^{\prime}$, we can remove $x$ additional elements from the list $S_{r}^{\prime}(w)$. Then, $\left\lceil\left|S_{r}^{\prime}(v)\right| / 2\right\rceil \leq \max (v)-\min (v)$. The same method can be applied to all vertices $v \in V$ for which $\left\lceil\left|S_{r}^{\prime}(v)\right| / 2\right\rceil>\max (v)-\min (v)$ and afterwards every second remaining element of $S_{r}^{\prime}(v)$ is added to the solution $P(v)$. At the end $\sum_{v \in V}|P(v)| \geq \frac{1}{3} \operatorname{ch}^{*}(I)$.

The next result shows that the $\min (u)$ requirements have a great impact on the computational complexity of the problem. 
Proposition 1 If $I$ is defined on a complete graph and $\max (v) \leq M$ for all $v$ then $\widetilde{\text { ch }}(I)$ can be approximated within a factor of 2 in time $O(M n|C| \sqrt{M n+|C|})$, where $|C|$ denotes the number of frequencies in the spectrum.

Proof. It can be easily seen that

$$
\frac{c h^{*}(I)}{2} \leq \widetilde{\operatorname{ch}}(I) \leq \operatorname{ch}^{*}(I)
$$

Namely, when there are no minimum channel requirements then one can construct a feasible solution having at least value $c h^{*}(I) / 2$ by taking every second element from an optimal solution that may violate the co-site constraints. Hence, the algorithm for $c h^{*}(I)$ can be used to approximate $\widetilde{\operatorname{ch}}(I)$.

Note that this reasoning remains valid when the condition $\min (u)=0$, for all $u$, is replaced by $\min (v) \leq 1$, for all $v$.

Now the question can be asked if the channel stability number of a complete graph is $(1-\epsilon)$-approximable for every rational $0<\epsilon<1$, when all minimum frequency requirements equal zero. Unfortunately, it follows from the next theorem that even in that case there is a constant $c>0$ such that there is no deterministic polynomial time algorithm for $\widetilde{c h}(I)$ with the performance guarantee $c$, provided that $P \neq N P$. Recall that the channel stability number for the special instances which do not have minimum requirements-i.e. $\min (u)=0$ for all $u$ - is denoted by $\widetilde{c h}$.

Theorem 3 Computing $\widetilde{c h}(I)$ is MAX SNP-hard.

The proof of Theorem 3 requires several steps. The first is to establish the MAX SNPhardness of a special class of MAX 3SAT instances. In [PY'91] it is proven that MAX 3SAT-B is MAX SNP-hard for any $B \geq 8$. In order to prove Theorem 3 we need to show that MAX 3SAT- $B$ remains MAX SNP-hard when the instances are restricted to $B=6$ and moreover have a special clause structure.

Definition 1 (MAX 3SAT*) Let $I$ be a set of clauses of length 3 or 2 such that each variable occurs in exactly 4,5 or 6 clauses. Moreover, if a variable $x_{i}$ occurs in 4 or 6 clauses then these are respectively 2 or 3 pairs of symmetric clauses of the form $x_{i} \vee \bar{x}_{j}$ and $\bar{x}_{i} \vee x_{j}$. If a variable $x_{i}$ appears in 5 clauses then these are two pairs of symmetric clauses and one arbitrary clause of length $3 . M A X 3 S A T^{*}$ problem is to find a maximum number of simultaneously satisfiable clauses in I.

A slight modification of the original MAX SNP-hardness proof of Papadimitriou and Yannakakis establishes the MAX SNP-hardness of MAX 3SAT*. The reader can refer to [PY'91] for more details.

Lemma 1 MAX $3 S A T^{*}$ is MAX SNP-hard.

\section{Proof:}

The only difference between the MAX 3SAT- $B$ instance obtained in Theorem 2(b) in [PY'91] and MAX 3SAT* is that in the first case there can be some variables occuring in four pairs of symmetric clauses. This can be eliminated in the following way. We use an $L$-reduction from MAX 3SAT similar to [PY'91]. For each variable occuring $m$ times in the 3SAT-formula 
one constructs a graph $F_{m}$ with the following properties: (1) it has degree bounded by 3 (in [PY'91] it was bounded by 4), (2) it has $O(m)$ nodes of which $m$ are 'distinguished', and (3) for every partition of the nodes into two sets, the number of edges in the cut is at least $\min \left(\left|S_{1}\right|,\left|S_{2}\right|\right)$, where $S_{1}$ and $S_{2}$ are the sets of distinguished nodes in the two sides. $F_{m}$ can be obtained from $m$ full binary trees with (at least) $1 / c$ leaves. The trees are connected in a slightly different way than in [PY'91]. Namely, let us denote the original leaves by $L$. We connect four additional nodes $v^{\prime}, v_{1}, v_{2}$ and $v_{3}$ to each $v \in L$ using the edges $\left(v, v^{\prime}\right),\left(v, v_{3}\right)$, $\left(v^{\prime}, v_{1}\right)$ and $\left(v^{\prime}, v_{2}\right)$. Now, consider a cubic $c$-expander ([A'87]) on the original leaves $L$ and for each of its edges $(v, w)$ introduce one edge between $v_{i}$ and $w_{j}$ for some $i, j \in\{1,2,3\}$ Altogether, each of the new leaves $\left\{v_{1}, v_{2}, v_{3} \mid v \in L\right\}$ should be used exactly once. The distinguished nodes are the roots of the binary trees. Then, clearly, $F_{m}$ satisfies properties (1) and (2). To show that the property 3 is satisfied a similar reasoning to [PY'91] can be used. Let us consider a partition of the nodes into two sets $Q_{1}$ and $Q_{2}$. Define $R_{1}$, and respectively $R_{2}$, to be the set of distinguished nodes for which all the inner nodes of the corresponding binary trees are contained in $Q_{1}$, or respectively in $Q_{2}$. Let us denote the rest of the distinguished nodes by $R_{3}$. The cut clearly contains at least $\left|R_{3}\right|$ edges joining inner nodes of the binary trees. We claim that it also contains $\min \left(\left|R_{1}\right|,\left|R_{2}\right|\right)$ edges incident to some leaves.

To see the latter, let us denote by $Q_{1}^{*}=Q_{1} \cap L$ and respectively by $Q_{2}^{*}=Q_{2} \cap L$. W.l.o.g. let us assume that $\left|Q_{1}^{*}\right| \leq\left|Q_{2}^{*}\right|$. Then $\left|Q_{1}^{*}\right| \geq \frac{1}{c} \min \left(\left|R_{1}\right|,\left|R_{2}\right|\right)$ and in a $c$-expander on nodes $L$ this set would have at least $c \cdot \frac{1}{c} \min \left(\left|R_{1}\right|,\left|R_{2}\right|\right)$ neighbors outside. For each such neighbor $w$ we can choose from the $c$-expander an adjacent node $v \in Q_{1}^{*}$. In the graph $F_{m}$ there is a path from $v$ to $w$ of the form: $v\left(v^{\prime}\right) v_{i} w_{j}\left(w^{\prime}\right) w, i, j \in\{1,2,3\}$. Notice that $v, v^{\prime} \in Q_{1}$ and $w \in Q_{2}$ and hence, at least one of the edges in the path belongs to the cut. Since for each $w$ we consider only one path and since paths starting in the vertex $v$ may overlap only in the edge $\left(v, v^{\prime}\right)$, which does not belong to the cut, we can altogether select at least $\min \left(\left|R_{1}\right|,\left|R_{2}\right|\right)$ different cut edges from these paths. Since none of these cut edges has two inner nodes as its ends, they are different from the first $\left|R_{3}\right|$ edges.

Now $\left|R_{3}\right|+\min \left(\left|R_{1}\right|,\left|R_{2}\right|\right) \geq \min \left(\left|S_{1}\right|,\left|S_{2}\right|\right)$ and $F_{m}$ satisfies (3). The rest of the construction and of the proof is the same as in [PY'91]. Each variable $x$ occurring $m$ times in the instance of MAX 3SAT is replaced by $O(m)$ new variables $x_{1}, x_{2}, \ldots$ Moreover, for every edge $(i, j)$ of the graph $F_{m}$ two auxiliary clauses are introduced $x_{i} \vee \bar{x}_{j}$ and $\bar{x}_{i} \vee x_{j}$. It can be now easily seen that this transformation from MAX 3SAT to MAX 3SAT* is an $L$-reduction.

We now exhibit an L-reduction from MAX $3 \mathrm{SAT}^{*}$ to $\widetilde{c h}(\cdot)$ when the underlying graph topology is that of complete graphs. We define a transformation $f$ from the MAX 3SAT* problem into the channel stability problem. If an instance $I$ of MAX 3SAT* has $m$ clauses and $n$ variables, then $I^{\prime}=f(I)$ is defined on a complete graph with $3 n+m$ vertices and the set of channels $C=\left\{x_{i}^{1}, \bar{x}_{i}^{1}, x_{i}^{2}, \bar{x}_{i}^{2}, x_{i}^{3}, \bar{x}_{i}^{3}, a_{i}, b_{i}, c_{i}, d_{i} \mid i=1, \ldots, n\right\}$. The first six channels represent the occurrences of a variable $x_{i}, i=1, \ldots, n$. Positive literals are represented by positive channels and negative literals by negative ones. The occurence in a clause of length 3 is always represented by the channel with upper index 1 . Two literals from a pair of a symmetric clauses are represented by two channels with the same upper index. For each variable $x_{i}$ we introduce 3 nodes $t_{i}, p_{i}$ and $r_{i}$ with lists of channels: $L\left(t_{i}\right)=$ $\left\{b_{i}, \bar{x}_{i}^{1}, a_{i}, \bar{x}_{i}^{2}, x_{i}^{3}, \bar{x}_{i}^{3}, x_{i}^{2}, c_{i}, x_{i}^{1}, d_{i}\right\}, L\left(p_{i}\right)=\left\{b_{i}, d_{i}\right\}$ and $L\left(r_{i}\right)=\left\{a_{i}, c_{i}\right\}$. The sequence $L\left(t_{i}\right)$ defines a linear order of consecutive channels. We define $\max \left(t_{i}\right)=5, \max \left(p_{i}\right)=\max \left(r_{i}\right)=$ 1. Intuitively, the task of group $t_{i}, r_{i}, p_{i}$ is to ensure that only two set assignments for $S\left(t_{i}\right)$, 
$S\left(p_{i}\right)$ and $S\left(r_{i}\right)$ reach the maximum requirements. For $S\left(t_{i}\right)$ it is either $\left\{b_{i}, a_{i}, x_{i}^{3}, x_{i}^{2}, x_{i}^{1}\right\}$ or $\left\{\bar{x}_{i}^{1}, \bar{x}_{i}^{2}, \bar{x}_{i}^{3}, c_{i}, d_{i}\right\}$. For each clause $c_{j}$ we introduce one node $k_{j}, j=1, \ldots, m$. With each such node we associate the set of channels representing the literals of the respective clause and set $\max \left(k_{j}\right)=1$. If a variable $i$ occurs in 5 clauses than either the channel $x_{i}^{1}$ or $\bar{x}_{i}^{1}$ is not admissible for any clause-node. If a variable $i$ occurs 4 times than both of these channels are absent in the lists of admissible channels for clause-nodes.

Let us illustrate this transformation by means of an example. Given a boolean formula $I$ of the form

$$
\left(x_{1} \vee \bar{x}_{2} \vee x_{3}\right) \wedge\left(x_{1} \vee \bar{x}_{2}\right) \wedge\left(\bar{x}_{1} \vee x_{2}\right) \wedge\left(x_{2} \vee \bar{x}_{3}\right) \wedge\left(\bar{x}_{2} \vee x_{3}\right) \wedge\left(x_{1} \vee \bar{x}_{3}\right) \wedge\left(\bar{x}_{1} \vee x_{3}\right)
$$

we obtain an instance $I^{\prime}$ of the channel stability problem defined on a complete graph $K_{16}$ with the following lists of admissible channels:

$$
\begin{array}{lll}
L\left(t_{i}\right)=\left\{b_{i}, \bar{x}_{i}^{1}, a_{i}, \bar{x}_{i}^{2}, x_{i}^{3}, \bar{x}_{i}^{3}, x_{i}^{2}, c_{i}, x_{i}^{1}, d_{i}\right\}, \quad i=1,2,3, \\
L\left(p_{i}\right)=\left\{b_{i}, d_{i}\right\}, & i=1,2,3, & \\
L\left(r_{i}\right)=\left\{a_{i}, c_{i}\right\}, & i=1,2,3, & \\
L\left(c_{1}\right)=\left\{x_{1}^{1}, \bar{x}_{2}^{1}, x_{3}^{1}\right\}, & & \\
L\left(c_{2}\right)=\left\{x_{1}^{2}, \bar{x}_{2}^{2}\right\}, & L\left(c_{3}\right)=\left\{\bar{x}_{1}^{2}, x_{2}^{2}\right\}, \\
L\left(c_{4}\right)=\left\{x_{2}^{3}, \bar{x}_{3}^{2}\right\}, & L\left(c_{5}\right)=\left\{\bar{x}_{2}^{3}, x_{3}^{2}\right\}, \\
L\left(c_{6}\right)=\left\{x_{1}^{3}, \bar{x}_{3}^{3}\right\}, & L\left(c_{7}\right)=\left\{\bar{x}_{1}^{3}, x_{3}^{3}\right\} . &
\end{array}
$$

A solution $S$ of such an instance of the channel stability problem is defined to be regular if and only if, for every $i \in\{1, \ldots, n\}$, (a) each $S\left(t_{i}\right)$ is either $\left\{b_{i}, a_{i}, x_{i}^{3}, x_{i}^{2}, x_{i}^{1}\right\}$ or $\left\{\bar{x}_{i}^{1}, \bar{x}_{i}^{2}, \bar{x}_{i}^{3}, c_{i}, d_{i}\right\}$; (b) no channel is assigned both positive and negated to some clausenodes, i.e. for no $x_{i}$ and indices $j, l, c, d, x_{i}^{j} \in S\left(k_{c}\right)$ and $\bar{x}_{i}^{l} \in S\left(k_{d}\right)$.

Any satisfying assignment induces, in the obvious way, a regular solution. Conversely, a regular solution defines a proper truth assignment for the original 3SAT formula. The difficulty of the proof is to show that any given feasible solution can be transformed into a regular one whose value is no worse than the original.

Lemma 2 If $I$ is an instance of the $M A X 3 S A T^{*}$ problem and $I^{\prime}=f(I)$ has a solution $S_{1}$ of value $c$, then $I^{\prime}$ has also a solution $S_{2}$ of value at least c satisfying the additional property:

$$
\neg\left(\exists i, \exists j \text { s.t. }\left\{x_{i}^{j}, \bar{x}_{i}^{j}\right\} \subseteq S\left(t_{i}\right)\right) .
$$

Proof. Such pairs of channels can be iteratively removed from the solution $S_{1}$ for $i=$ $1, \ldots, n$ and $j=1,2,3$ without decreasing the value of the solution:

- $(\mathrm{j}=1)$ If $\left.\left\{x_{i}^{1}, \bar{x}_{i}^{1}\right\} \subseteq S\left(t_{i}\right)\right)$ then $\left|S\left(t_{i}\right)\right| \leq 4$. Moreover, $b_{i} \notin S\left(t_{i}\right)$ and $d_{i} \notin S\left(t_{i}\right)$. Since $S\left(p_{i}\right)$ can contain only one of the colors $b_{i}$ and $d_{i}$, the other one can replace $x_{i}^{1}$ or $\bar{x}_{i}^{1}$ in $S\left(t_{i}\right)$. The value of the modified solution does not change.

- $(\mathrm{j}=2)$ If $\left.\left\{x_{i}^{2}, \bar{x}_{i}^{2}\right\} \subseteq S\left(t_{i}\right)\right)$ then $\left|S\left(t_{i}\right)\right| \leq 4$ and none of the channels: $a_{i}, x_{i}^{3}, \bar{x}_{i}^{3}, c_{i}$ is in $S\left(t_{i}\right)$. Moreover, we can now assume that only one of the channels $x_{i}^{1}$ and $\bar{x}_{i}^{1}$ belongs to $S\left(t_{i}\right)$. If $x_{i}^{1} \notin S\left(t_{i}\right)$ then $x_{i}^{2}$ can be replaced in $S\left(t_{i}\right)$ by $c_{i}$ and $S\left(r_{i}\right)$ can be set to $\left\{a_{i}\right\}$. Otherwise, if $\bar{x}_{i}^{1} \notin S\left(t_{i}\right)$ then $\bar{x}_{i}^{2}$ is replaced in $S\left(t_{i}\right)$ by $a_{i}$ and $S\left(r_{i}\right)$ is set to $\left\{x_{i}^{c}\right\}$. The value of the modified solution does not decrease.

- $(\mathrm{j}=3)$ Since $x_{i}^{3}$ and $\bar{x}_{i}^{3}$ are consecutive they could not have been together chosen for $S\left(t_{i}\right)$. 
Lemma 3 If $I$ is an instance of the $M A X 3 S A T^{*}$ problem and $I^{\prime}=f(I)$ has a solution $S_{1}$ of value $c$, then $I^{\prime}$ has also a solution $S_{2}$ of value at least $c$ satisfying the additional property:

$$
\neg\left(\exists i, \exists j \text { and two clause-nodes } k_{k} \text { and } k_{l} \text { s.t. } x_{i}^{j} \in S\left(k_{k}\right) \text { and } \bar{x}_{i}^{j} \in S\left(k_{l}\right)\right) .
$$

Proof. Note that if $x_{i}^{j} \in S\left(k_{k}\right)$ and $\bar{x}_{i}^{j} \in S\left(k_{l}\right)$ for some $i \in\{1, \ldots, n\}, j \in\{1,2,3\}$ then $k_{k}$ and $k_{l}$ must correspond to a pair of symmetric clauses of the form $x_{i}^{j} \vee \bar{x}_{s}^{u}$ and $\bar{x}_{i}^{j} \vee x_{s}^{u}$. By Lemma 2 we can assume that either $\bar{x}_{s}^{u}$ or $x_{s}^{u}$ does not belong to $S\left(t_{s}\right)$. If $x_{s}^{u} \notin S\left(t_{s}\right)$, then we can set $S\left(k_{l}\right)=\left\{x_{s}^{u}\right\}$ instead of $\left\{\bar{x}_{i}^{j}\right\}$. Similarly, if $\bar{x}_{s}^{u} \notin S\left(t_{s}\right)$, then we can set $S\left(k_{k}\right)=\left\{\bar{x}_{s}^{u}\right\}$. The value of the modified solution $S_{2}$ equals the value of the original solution $S_{1}$.

Lemma 4 If $I$ is an instance of the MAX $3 S A T^{*}$ problem and $I^{\prime}=f(I)$ has a solution $S$ such that $\left|S\left(t_{i}\right) \cup S\left(p_{i}\right) \cup S\left(r_{i}\right)\right|=7$, for any $i=1, \ldots, n$, then either only positive or only negative channels corresponding to the variable $x_{i}$ are chosen to the sets $S\left(k_{l}\right)$, where $k_{l}$ represents a clause, $l=1, \ldots, m$.

Proof. If $\left|S\left(t_{i}\right) \cup S\left(p_{i}\right) \cup S\left(r_{i}\right)\right|=7$ then $\left|S\left(t_{i}\right)\right|=5,\left|S\left(p_{i}\right)\right|=1$ and $\left|S\left(r_{i}\right)\right|=1$. Then, the only two possibilities for $S\left(t_{i}\right)$ are $\left\{\bar{x}_{i}^{1}, \bar{x}_{i}^{2}, \bar{x}_{i}^{3}, c_{i}, d_{i}\right\}$ and $\left\{b_{i}, a_{i}, x_{i}^{3}, x_{i}^{2}, x_{i}^{1}\right\}$. Hence, all the channels representing the variable $x_{i}$ that are chosen for some clause-nodes are either positive or negative.

Lemma 5 If $I$ is an instance of the $M A X 3 S A T^{*}$ problem and $I^{\prime}=f(I)$ has a solution $S_{1}$ of value $c$, then $I^{\prime}$ has also a regular solution $S_{2}$ of value not less than $c$.

Proof. By Lemma 3 we can assume that for any $i=1, \ldots, n$ maximally three channels corresponding to the variable $x_{i}$ are chosen to the sets $S\left(k_{l}\right)$, where $k_{l}$ represents a clause and $l=1, \ldots, m$. Hence, the requirements that either only positive channels or only negative channels are chosen for the clause-nodes can be violated at most by one channel. W.l.o.g. assume that one negative and two positive channels corresponding to the variable $x_{i}$ are selected for some clause-nodes. In that case, by Lemma $4,\left|S\left(t_{i}\right) \cup S\left(p_{i}\right) \cup S\left(r_{i}\right)\right|<7$. Hence, instead of assigning the negative channel to the clause-node, we can add it to $S\left(t_{i}\right)$. More precisely, $S\left(t_{i}\right)$ can be set to $\left\{\bar{x}_{i}^{1}, \bar{x}_{i}^{2}, \bar{x}_{i}^{3}, c_{i}, d_{i}\right\}, S\left(p_{i}\right)=\left\{b_{i}\right\}$ and $S\left(r_{i}\right)=\left\{a_{i}\right\}$. By this modification the value of the solution does not decrease.

\section{Proof of Theorem 3.}

We claim that the function $f$ defined above is an $L$-reduction of the MAX 3SAT* problem into the channel stability problem in complete graphs. Clearly, for any instance $I$ and $I^{\prime}=f(I)$, we have $O P T\left(I^{\prime}\right) \geq O P T(I)+7 n$. On the other hand, by Lemma 5 each solution of $I^{\prime}$ of value $7 n+k$ can be made regular. Then, defining the truth assignment in $I$ according to the channels selected for the clause-nodes yields a solution for $I$ of value $k$. Therefore, $O P T\left(I^{\prime}\right) \leq O P T(I)+7 n$. For any instance $I$ of MAX $3 \mathrm{SAT}^{*}$ we have $4 n \leq 3 m$ and $O P T(I) \geq \frac{m}{2}$. It follows that $O P T\left(I^{\prime}\right) \leq \frac{23}{2} O P T(I)$ and $|k-O P T(I)|=\left|7 n+k-O P T\left(I^{\prime}\right)\right|$. Hence, $f$ is an $L$-reduction, where the constant $\alpha$ can be set to $\frac{23}{2}$ and $\beta=1$. 


\section{Sparse lists of admissible channels}

The channel stability number of complete graphs can be easily computed when the co-site constraints can be ignored, but the problem becomes NP-hard when the constraints have to be observed. It would be interesting to know if this difference disappear when the lists of admissible channels satisfy certain additional conditions. As a partial answer to this question we first consider instances with relatively short lists of channels and show how the approximation results can be improved when $|L(v)|<2 \max (v)$ for every $v \in V$. In Section 5 some conditions for dense lists of admissible channels are defined, under which the co-site constraints have no influence on the value of an optimal solution.

The approximation ratio can be improved using the notion of capacity. We mean the capacity of lists of admissible channels for some vertices $A \subseteq V i$. e. the number of channels that can be possibly assigned to these vertices respecting the co-site constraints.

Definition 2 Given a set of linearly ordered channels $C=\{1, \ldots,|C|\}$ and a complete graph $K_{n}$ with lists of admissible channels $L(v)$ for every vertex $v \in V\left(K_{n}\right)$, the capacity $(A)$, $A \subseteq V\left(K_{n}\right)$, is defined as the maximum value of the sum $\sum_{v \in V}|S(v)|$ subject to the condition that $S(v) \subseteq L(v)$ and $S(v) \cap S(u)=\emptyset$ for all $v, u \in V\left(K_{n}\right)$ as well as to the co-site constraints.

Note that in this definition there is no bound on the cardinality of $S(v)$. The usefulness of the capacity notion comes from the fact that it can be quickly computed for any set of vertices $A \subseteq V\left(K_{n}\right)$ and then used to approximate the value of $\operatorname{ch}(I)$. For any instance $I$ of the channel stability problem let us construct an auxiliary digraph $G=(W, F)$. It has one vertex $v_{c}$ for every vertex $v \in A$ and every channel $c$ such that $c \in L(v)$ and an arc $\left(v_{c}, w_{c+1}\right)$, for all $w \neq v$. The interpretation of this arc is that if channel $c$ is assigned to the vertex $v$ then $c+1$ can be assigned to $w$. Let $\mathcal{P}$ be a family of vertex-disjoint paths in $G$ having the property that for every channel $c$ there is at most one vertex $v_{c}$ contained in any of the paths $\mathcal{P}$. By the definition of the digraph $G$ the family $\mathcal{P}$ corresponds to an assignments of channels to vertices fullfiling the requirements from Defintion 2. Therefore,

$$
\operatorname{capacity}(A)=\max _{\mathcal{P}}\left\{\sum_{P \in \mathcal{P}}|P|\right\} .
$$

The family $\mathcal{P}$ maximizing the above expression can be found using a kind of BFS. Let $c_{0}$ be the lowest frequency in the available spectrum. Then, for all $v \in V$ such that $c_{0} \in L(v)$, the nodes $v_{c_{0}}$ are put into the BFS queue. Now, a longest directed path starting in any of these nodes is computed. Assume that this path ends in $w_{c}$. It means that channel $c$ could be assigned to vertex $w$ and that channel $c+1$ does not occur in any list $L(v), v \in A$ and $v \neq w$. Hence, the next channel that could be assigned to any vertex must be higher than $c+1$. Therefore, the second path can start in any of the nodes $v_{c+a}$, for the smallest channel $c+a>c+1$ that is admissible for any vertex. The procedure continues in the same way until the last channel is reached.

If the lists of admissible channels are short then the next theorem gives a better performance ratio than Proposition 1.

Theorem 4 Assume that we are given an instance $I$ with a set of linearly ordered channels $C=\{1, \ldots,|C|\}$ and a complete graph $K_{n}$ with lists of admissible channels $L(v)$ and maximum channel requirements $\max (v)$ for every vertex $v \in V\left(K_{n}\right)$. If there is a constant 
$\alpha$ such that for every vertex $v \in V\left(K_{n}\right)$

$$
\frac{\operatorname{capacity}(v)}{\max (v)} \leq \alpha
$$

then $\frac{\operatorname{capacity}(V)}{\alpha} \leq \widetilde{\operatorname{ch}}(I) \leq \operatorname{capacity}(V)$.

\section{Proof:}

Consider a family of disjoint paths $\mathcal{P}$ defined as above and covering capacity $(V)$ nodes. Such a family induces an assignment $S(v)$ of channels to vertices:

$$
S(v):=\left\{c: \exists P \in \mathcal{P} \text { s.t. } v_{c} \in P\right\}
$$

Note that $|S(v)| \leq \operatorname{capacity}(v)$, for every vertex $v \in V\left(K_{n}\right)$. In a proper assignment no selected sublist $S(v)$ should be longer than $\max (v)$ and therefore, all longer lists $S(v)$ have to be reduced. However, for each $v$, at most capacity $(v)-\max (v)$ channels are removed which, in the worst case, constitutes a $\left(1-\frac{1}{\alpha}\right)$ fraction of the number of channels assigned to $v$. The remaining channels form a proper assignment at least of value $\operatorname{capacity}(V) / \alpha$.

\section{Dense lists of admissible channels}

In this section we go back to the study of $\operatorname{ch}(\cdot)$, namely we consider again constraints (a) through (c). We examine certain "density" conditions on the input lists $L(v)$ 's, which guarantee that the value of optimal solutions does not depend on the co-site constraints.

The study of "dense" lists of admissible channels is motivated by the results obtained for randomly generated instances of the channel stability problem in complete graphs. We have generated 10 instances with 15 vertices, 60 channels and the average number of 30 forbidden channels for each vertex. Minimum channel requirements have been set to 1 and maximum requirements have been equal 4 or 6 . In 9 cases a heuristic algorithm managed to assign all colors to the vertices observing both the minimum and maximum requirements as well as the co-site constraints.

The following theorem formalizes the notion of "density".

Theorem 5 Assume that we are given an instance $I$ with a set of linearly ordered channels $C=\{1, \ldots,|C|\}$, a complete graph $K_{n}$ with lists of admissible channels $L(v)$ and minimum and maximum channel requirements $\min (v)$ and $\max (v)$ for every vertex $v \in V\left(K_{n}\right)$. Moreover, assume that there is a constant $k<n-1$ such that the instance has the following two properties:

(1) $\forall A \subseteq V\left(K_{n}\right)\left(|A| \leq k+1 \Rightarrow\left|\bigcup_{v \in A} L(v)\right|>4 \sum_{v \in A} \max (v)\right)$

(2) $\forall A \subseteq V\left(K_{n}\right)\left(|A|=k \Rightarrow \bigcup_{v \in A} L(v)=C\right)$.

Then the value of an optimal solution of the channel stability problem is the same when the co-site constraints are observed and when they are ignored. Moreover, a feasible solution exists iff $\sum_{v \in V} \min (v) \leq|C|$ and then

$$
\operatorname{ch}(I)=\min \left\{|C|, \sum_{v \in V} \max (v)\right\} .
$$


Proof. Given any solution $S$ of an instance $I$ of the channel stability problem with minimum requirements we call a channel $c$ to be free for a vertex $v$ iff none of the channels $\{c-1, c, c+1\}$ has been chosen to $S(v)$. A channel $c$ is used by a vertex $v$ iff $c \in S(v)$.

Let us first point out some consequences of the properties (1) and (2). It follows from the first property that for any set of vertices $A,|A| \leq k+1$, the colors from the set $\bigcup_{v \in A} L(v)$ can be partitioned into disjoint groups of $4 \max (v)$ colors per vertex, so that only colors from the list $L(v)$ are in the group associated with any vertex $v, v \in A$. For one vertex one more color can be selected. In a proper assignment $S$ satisfying all requirements, the selection of a channel $c$ for a vertex $v$ may cause that maximally three admissible channels: $c-1, c$ and $c+1$ are not free for $v$. Hence, given any admissible assignment $S$ and a set of vertices $A,|A| \leq k+1$, the number of free channels in $A$ exceeds at least by one the number of used channels. The property (2) guarantees that for every channel $c$ and an arbitrary set of vertices $A$ of cardinality $k+2$ there are at least three vertices $u \in A$ such that $c \in L(u)$. Hence, for any admissible assignment $S$, if colors $c$ and $c+1$ or $c-1$ have been chosen to $S(v)$ for any vertex $v \in A$ then $c$ is free for at least one vertex $w \in A$.

Clearly, if $\sum_{v \in V} \min (v) \leq|C|$ then there is no feasible solution. Otherwise, it follows from the Hall property (see e.g. [B'85]) that $\min \left\{|C|, \sum_{v \in V} \max (v)\right\}$ channels can be assigned to the vertices when the co-site constraints are ignored. Moreover, we claim that all violations of the co-site constraints can be removed from such an optimal assignment $S$. Namely, assume that there is a vertex $v$ that has been assigned two consecutive channels $c$ and $c+1$. Then $S$ can be modified to a solution $S^{\prime}$ that has the same value, assigns no new pairs of consecutive channels and such that $c \notin S(v)$. This is achieved with the help of an auxiliary directed tree $T$ with labeled edges that is iteratively augmented until the new solution $S^{\prime}$ is found. The vertex $v$ is defined to be the root of $T$. Then, new nodes are added to $T$ according to the following principle. If the channel $c$ is free for the vertex $w$ that has been just added to $T$ or if there is a channel $d$ that is free for $w$ and is not used by any other vertex than the unique path from $v$ to $w$ defines the sought transformation from $S$ to $S^{\prime}$. Namely, in the first case the channel $c$ is assigned to $w$ instead of the channel that labeled the last arc of the path and in the second case $d$ is allocated to $w$. Moreover, for every arc $\left(v_{1}, v_{2}\right)$ in the path labeled with $c^{*}$, the channel $c^{*}$ is removed from the list of channels assigned to $v_{2}$ and is now allocated to $v_{1}$. By the second property when $|V(T)|=k+2$ then $c$ is free for at least one vertex in $V(T)$ and hence, the transformation from $S$ to $S^{\prime}$ is found. Otherwise, if $|V(T)|<k+2$ and none of the two cases applies then by the first property there is a vertex $u \in V(T)$ and a channel $f$ such that $f$ is free for $u$ and is not used by any of the vertices in $T$. Then, there is a vertex $z \notin V(T)$, such that $f \in S(z)$. The tree $T$ is now extended by the arc $(u, z)$ labeled $f$. Since in each such step a new vertex is added to $V(T)$ and $k \leq n-2$ the sought transformation is found at most after $k+1$ steps. Moreover, the same method can be iteratively applied to all pairs of consecutive channels that are assigned to one vertex and at the end we obtain a solution satisfying all constraints and assigning $\min \left\{|C|, \sum_{v \in V} \max (v)\right\}$ channels.

The requirements in Theorem 5 are quite restrictive and therefore, it would be good to know if there are some lighter conditions on the lists of admissible channels under which the co-site constraints have no impact on the channel stability number $\operatorname{ch}(\cdot)$. However, the following two examples show that none of the properties 1 and 2 alone can be sufficient.

Example 1. Consider a complete graph $K_{n}$ with vertices $\left\{v_{1}, \ldots, v_{n}\right\}$. For the vertices $v_{i}, i=1, \ldots n-1$ the lists of admissible channels are defined as $L\left(v_{i}\right)=\{1,2, \ldots, n-1\}$ and maximum channel requirements are $\max (v)=1$. For the last vertex $v_{n}, L\left(v_{n}\right)=$ 
$\{1,2, \ldots, n, n+1\}$ and $\max \left(v_{n}\right)=2$. The minimum channel requirements of all vertices equal zero. Then $\operatorname{ch}(I)=n$ but an optimal solution that does not observe the co-site constraints achieves value $n+1$. On the other hand, this instance satisfies the first property from Theorem 5 for any constant $k<\left\lfloor\frac{n}{4}\right\rfloor-1$.

Example 2. In this example we also consider $n+1$ channels and a complete graph $K_{n}$. For the first $n-1$ vertices the lists of admissible channels are defined as $L\left(v_{i}\right)=\{1,2, \ldots, n+1\}$ and $\max \left(v_{i}\right)=1$. For the last vertex $v_{n}$ we set $L\left(v_{n}\right)=\{1,2\}$ and $\max \left(v_{n}\right)=2$. Then, as in the previous example, $\operatorname{ch}(I)=n$ and an optimal solution that does not observe the co-site constraints achieves value $n+1$. However, the second property from Theorem 5 is satisfied for every subset of vertices $A,|A|>2$.

\section{Conclusions}

In this work we have studied the channel stability function $\operatorname{ch}(I)$ of an instance $I$ defined on a graph $G$. This function can be used to select a channel assignment plan for the fixed part of a mixed cellular network leaving enough freedom in the dynamic part. In particular we have studied the computational complexity and approximability of $\operatorname{ch}(I)$ when $G$ is a complete graph and the restriction of $\operatorname{ch}(I)$ to $\widetilde{c h}(I)$ when there are no minimum channel requirements. It has been shown that $\widetilde{c h}(I)$ can be approximated up to a constant factor but unfortunately the problem is still MAX SNP-hard. Moreover, the influence of the co-site constraints on the complexity of $\widetilde{c h}(I)$ and $\operatorname{ch}(I)$ has been examined.

The future work should concentrate on the design of algorithms for the general topology of the dynamic part of the network. The results for complete graphs can be used in the computation of upper bounds on $\operatorname{ch}(I)$. One possibility is to find an appropriate disjoint clique cover of $G$ and then sum up known bounds for the cliques. This idea is now being implemented and the results will be compared with lower bounds obtained by heuristic algorithms based on frequency assignment procedures.

Another approach to the evaluation of lists of admissible channels in the dynamic part of the network could be based on the comparison of the channel stability number for different maximal cliques of $G$. In that case it remains to be examined how the values for single cliques should be combined together into a function characterizing the whole graph.

It would also be interesting to study the complexity of the channel stability number when the frequency spectrum is independent of the input.

\section{References}

[A'87] M.Ajtai. Recursive construction for 3-regular expanders. Proc. 28th Annual IEEE Symp. on Foundations of Computer Science (1987), 295-304.

[A'94] S. Arora. Probabilistic checking of proofs and the hardness of approximation problems. PhD Thesis, U.C. Berkeley, 1994. Available via anonymous ftp as Princeton TR94-476.

[BGS'93] M. Bellare, O. Goldreich, and M. Sudan. Free bits, PCPs and nonapproximability - towards tight results. Technical Report ECCC TR95-24, Revised version, September 1995. Extended abstract in Proc. 25th ACM Symp. on Theory of Computing (1993), pp. 113-131, 1993. 
[B'85] C. Berge. Graphs. North-Holland Math. Library, Vol. 6, Part 1, Elsevier Science Publishers (1985).

[B'89] C. Berge. Minimax relations for the partial $q$-colorings of a graph. Disc. Mathematics 7/4 (1989), 3-14.

[CLR'90] T.H.Cormen, C.E.Leiserson and R.L.Rivest. Introduction to Algorithms. The MIT Press, Cambridge, McGraw Hill, 1990.

[DJLS'94] G. Dahl, K. Jörnsten, G. Løvnes, S. Svaet. Graph optimization problems in connection with the management of mobile communication systems. Telecommunications Systems, Vol. 3 (1994), 319-340.

[DV'93] D. Dimitrijević, J. Vučetić. Design and Performance Analysis of the Algorithms for Channel Allocation in Cellular Networks. IEEE Transactions on Vehicular Technology, Vol. 42 (1993), 526-534.

[GSW'94] A. Gräf, M. Stumpf, G. Weißenfels. On coloring unit disk graphs. Johannes Gutenberg-Universit" at Mainz (1994).

[EB'91] H. Eriksson, R. Bownds. Performance of Dynamic Channel Allocation in the DECT System. 41st IEEE Vehicular Technology Conference (1991), 693-698.

[H'80] W.K. Hale. Frequency Assignment: Theory and Applications. Proc. of the IEEE, Vol. 68 (1980), 1497-1514.

[H'94] J. Håstad. Recent results in hardness of approximation. Proc. of 3rd Scandinavian Workshop on Algorithm Theory (1994), Springer-Verlag LNCS 824, pp. 231-239.

[JT'95] T. Jensen, B. Toft. Graph Coloring Problems. John Wiley \& Sons, Inc., 1995.

[M'95] E. Malesińska. An Optimization Method for the Channel Assignment in Mixed Environments. Proc. of the 1st ACM Int. Conf. on Mobile Computing and Networking (1995), 210-217.

[MBHRR'95] M.V. Marathe, H. Breu, H.B. Hunt III, S.S. Ravi, D.J. Rosenkrantz. Simple Heuristics for Unit Disk Graphs. Networks, Vol.25 (1995), 59-68.

[PY'91] Ch.H. Papadimitriou, M. Yannakakis. Optimization, Approximation, and Complexity Classes. Journal of Computer and System Sciences 43 (1991), 425-440.

[P'95] J. Plehn. Private communication.

[R'92] G. Riva. Performance Analysis of an Improved Dynamic Channel Allocation Scheme for Cellular Mobile Radio Systems. 42nd IEEE Vehicular Technology Conference (1992), 794-797.

[T'89] B.A. Tesman. T-colorings, list T-colorings, and set T-colorings of graphs. RUTCOR Res. Rept. RRR 57-89 Rutgers University, New Brunswick, NJ (1989). 
[ZE'93] J. Zander, H. Eriksson. Asymptotic Bounds on the Performance of a Class of Dynamic Channel Assignment Algorithms. Wireless communications: future directions, ed. by J.M. Holtzman, D.J. Goodman, Kluwer Ac. Publ. (1993), 259-274. 
Reports from the group

\section{"Algorithmic Discrete Mathematics"}

of the Department of Mathematics, TU Berlin

498/1996 Ewa Malesinska, Alessandro Panconesi: On the Hardness of Allocating Frequencies for Hybrid Networks

496/1996 Jörg Rambau: Triangulations of Cyclic Polytopes and higher Bruhat Orders

483/1995 Rolf H. Möhring and Markus W. Schäffter: Approximation Algorithms for Scheduling Series-Parallel Orders Subject to Unit Time Communication Delays

478/1995 Sven G. Bartels: The complexity of Yamnitsky and Levin's simplices algorithm

477/1995 Jens Gustedt, Michel Morvan and Laurent Viennot: A compact data structure and parallel algorithms for permutation graphs, to appear in : Nagl et al., editors, Graph-Theoretic Concepts in Computer Science, Proccedings of the 20th International Workshop WG' 95.

476/1995 Jens Gustedt: Efficient Union-Find for Planar Graphs and other Sparse Graph Classes

475/1995 Ross McConnell and Jeremy Spinrad: Modular decomposition and transitive orientation

474/1995 Andreas S. Schulz: Scheduling to Minimize Total Weighted Completion Time: Performance Guarantees of LP-Based Heuristics and Lower Bounds

473/1995 Günter M. Ziegler: Shelling Polyhedral 3-Balls and 4-Polytopes

472/1995 Martin Henk, Jürgen Richter-Gebert and Günter M. Ziegler: Basic Properties of Convex Polytopes

471/1995 Jürgen Richter-Gebert and Günter M. Ziegler: Oriented Matroids

465/1995 Ulrich Betke and Martin Henk: Finite Packings of Spheres

463/1995 Ulrich Hund: Every simplicial polytope with at most $d+4$ vertices is a quotient of a neighborly polytope

462/1995 Markus W. Schäffter: Scheduling with Forbidden Sets

461/1995 Markus W. Schäffter: Drawing Graphs on Rectangular Grids with at most 2 Bends per Edge

458/1995 Ewa Malesińska: List Coloring and Optimization Criteria for a Channel Assignment Problem

447/1995 Martin Henk: Minkowski's second theorem on successive minima 
441/1995 Andreas S. Schulz, Robert Weismantel, Günter M. Ziegler: 0/1-Integer Programming: Optimization and Augmentation are Equivalent, appeared in Paul Spirakis (ed.): Algorithms - ESA '95, Lecture Notes in Computer Science 979, Springer: Berlin, 1995, pp. 473-483

440/1995 Maurice Queyranne, Andreas S. Schulz: Scheduling Unit Jobs with Compatible Release Dates on Parallel Machines with Nonstationary Speeds, appeared in Egon Balas and Jens Clausen (eds.): Integer Programming and Combinatorial Optimization, Lecture Notes in Computer Science 920, Springer: Berlin, 1995, pp. 307-320

439/1995 Rolf H. Möhring, Matthias Müller-Hannemann: Cardinality Matching: Heuristic Search for Augmenting Paths

436/1995 Andreas Parra, Petra Scheffler: Treewidth Equals Bandwidth for AT-Free ClawFree Graphs

432/1995 Volkmar Welker, Günter M. Ziegler, Rade T. Živaljević: Comparison Lemmas and Applications for Diagrams of Spaces

431/1995 Jürgen Richter-Gebert, Günter M. Ziegler: Realization Spaces of 4-Polytopes are Universal, to appear in Bulletin of the American Mathematical Society, October 1995.

430/1995 Martin Henk: Note on Shortest and Nearest Lattice Vectors

429/1995 Jörg Rambau, Günter M. Ziegler: Projections of Polytopes and the Generalized Baues Conjecture

428/1995 David B. Massey, Rodica Simion, Richard P. Stanley, Dirk Vertigan, Dominic J. A. Welsh, Günter M. Ziegler: Lê Numbers of Arrangements and Matroid Identities

408/1994 Maurice Queyranne, Andreas S. Schulz: Polyhedral Approaches to Machine Scheduling

407/1994 Andreas Parra, Petra Scheffler: How to Use the Minimal Separators of a Graph for Its Chordal Triangulation

401/1994 Rudolf Müller, Andreas S. Schulz: The Interval Order Polytope of a Digraph, appeared in Egon Balas and Jens Clausen (eds.): Integer Programming and Combinatorial Optimization, Lecture Notes in Computer Science 920, Springer: Berlin, 1995, pp. 50-64

396/1994 Petra Scheffler: A Practical Linear Time Algorithm for Disjoint Paths in Graphs with Bounded Tree-width

394/1994 Jens Gustedt: The General Two-Path Problem in time $O(m \log n)$, extended abstract

393/1994 Maurice Queyranne: A Combinatorial Algorithm for Minimizing Symmetric Submodular Functions

392/1994 Andreas Parra: Triangulating Multitolerance Graphs 
390/1994 Karsten Weihe: Maximum $(s, t)$-Flows in Planar Networks in $\mathcal{O}(|V| \log |V|)$ Time

386/1994 Annelie von Arnim, Andreas S. Schulz: Facets of the Generalized Permutahedron of a Poset, to appear in Discrete Applied Mathematics

383/1994 Karsten Weihe: Kurzeinführung in $\mathrm{C}++$

377/1994 Rolf H. Möhring, Matthias Müller-Hannemann, Karsten Weihe: Using Network Flows for Surface Modeling

376/1994 Valeska Naumann: Measuring the Distance to Series-Parallelity by Path Expressions

375/1994 Christophe Fiorio, Jens Gustedt: Two Linear Time Union-Find Strategies for Image Processing

374/1994 Karsten Weihe: Edge-Disjoint $(s, t)$-Paths in Undirected Planar graphs in Linear Time

373/1994 Andreas S. Schulz: A Note on the Permutahedron of Series-Parallel Posets, appeared in Discrete Applied Mathematics 57 (1995), pp. 85-90

371/1994 Heike Ripphausen-Lipa, Dorothea Wagner, Karsten Weihe: Efficient Algorithms for Disjoint Paths in Planar Graphs

Reports may be requested from: S. Marcus

Fachbereich Mathematik, MA 6-1

TU Berlin

Straße des 17. Juni 136

D-10623 Berlin - Germany

e-mail: Marcus@math.TU-Berlin.DE

Reports are available via anonymous ftp from: ftp.math.tu-berlin.de cd pub/Preprints/combi

file Report $-<$ number $>-<$ year $>$.ps.Z 